\title{
Investigação numérica da descontinuidade geométrica entre a entrada e a saída de um conversor de energia das ondas do mar do tipo coluna de água oscilante
}

\author{
Numerical investigation of geometrical discontinuity between input and output of an energy \\ converter of the sea waves of the oscillating water column type \\ M. N. Gomes ${ }^{1 *}$; Y. T. B. Lima²; L. A. O. Rocha ${ }^{3}$; E. D. Santos ${ }^{4}$; L. A. Isoldi ${ }^{4}$ \\ ${ }^{1}$ Intituto Federal do Paraná, Campus Paranaguá, 83215-750, Paranaguá-PR, Brasil \\ ${ }^{2}$ Programa de Pós-Graduação em Engenharia Mecânica - PROMEC, Universidade Federal do Rio Grande do Sul, \\ 90050-170, Porto Alegre-RS, Brasil \\ ${ }^{3}$ Departamento de Engenharia Mecânica, Universidade do Vale do Rio dos Sinos (UNISINOS), 93022-750, São \\ Leopoldo-RS, Brasil \\ ${ }^{4}$ Escola de Engenharia, Universidade Federal do Rio Grande, 96201-900, Rio Grande-RS, Brasil
}

*mateus.gomes@ifpr.edu.br

\begin{abstract}
A investigação realizada apresenta um estudo bidimensional de um dispositivo conversor de energia das ondas do mar do tipo Coluna de Água Oscilante (CAO) avaliado de forma numérica. Foram analisados três graus de liberdade $H_{1} / L$ (razão entre a altura e o comprimento da câmara $\mathrm{CAO}$ ), $H_{3}$ (profundidade de submersão do dispositivo CAO) e $L /$ (relação entre largura da câmara e da chaminé do dispositivo), no qual foi avaliado o efeito da variação geométrica no efeito de pistão, vazão mássica e pressão transiente para descontinuidade geométrica. A metodologia utilizada para avaliação numérica se fez através da utilização de um código comercial de dinâmica dos fluidos computacional, baseado no Método de Volumes Finitos (MVF). Como o trabalho apresenta interação entre dois fluidos imiscíveis (água e ar), utiliza-se o modelo multifásico Volume of Fluid (VOF). Os resultados obtidos mostram que à medida que $L / l$ diminui (por consequência a descontinuidade geométrica tende a diminuir) os picos negativos de pressão deixam de existir e a distribuição de pressões tende a ficar simétrica, e isso ocorre sempre quando $H_{l} / L$ tende a aumentar.

Palavras-chave: Energia das Ondas do Mar, Coluna de Água Oscilante, Descontinuidade Geométrica
\end{abstract}

The research carried out presents a two-dimensional numerical study of a numerically evaluated Oscillating Water Column (OWC) sea wave energy converter. Three degrees of freedom $H_{l} / L$ (ratio between height and length), $H_{3}$ (depth of immersion of the OWC device) and $L / l$ (relation between the width of the chamber and the chimney of the device) were analyzed, the effect of piston, mass flow and transient pressure for geometric discontinuity was evaluated. The methodology used for numerical evaluation was done through the use of a commercial code of computational fluid dynamics, based on the Finite Volume Method (FVM). As the work presents interaction between two immiscible fluids (water and air), the Multiphase Volume of Fluid (VOF) model is used. The results obtained show that as $L / l$ decreases (consequently the geometric discontinuity tends to decrease) the negative pressure peaks cease to exist and the pressure distribution tends to be symmetrical, and this occurs whenever $H_{l} / L$ tends to increase.

Keywords: Wave Energy, Oscillating Water Column, Geometrical Discontinuity

\section{INTRODUÇÃO}

À medida que o consumo energético mundial aumenta questões referentes ao ecossistema, energias renováveis e qualidade de vida levam ao estudo de novas formas sustentáveis de geração energética [1]. Pode-se considerar o oceano como uma das maiores fontes de energia renovável presente, tendo diferentes formas de extrai-la.

Em Tolmasquim (2016) [2], o autor apresenta dados referentes aos oceanos, em que, os mesmos cobrem aproximadamente $70 \%$ da superfície terrestre, sendo assim um provedor energético [2]. A energia contida nos mesmos pode ter diferentes classificações, conforme seu local de instalação e princípio de funcionamento $[2,3]$. 
Entre os vários conversores de energia de ondas, os dispositivos do tipo coluna de água oscilante (CAO) são os que se apresentam em maior escala de implementação e desenvolvimento em todo o mundo [4]. Os dispositivos CAO são considerados vantajosos em relação a muitos outros existentes pois nenhuma parte móvel está localizada na água [5].

A investigação da otimização geométrica de dispositivos conversores de energia das ondas do mar é um tema de pesquisa que permeia muitos trabalhos científicos dentre eles destacam-se alguns autores brasileiros como Gomes (2014) [6], Barbosa (2016) [7] e Martins (2016) [8]. Trabalhos internacionais também já foram realizados, dentre eles destacam-se Vyzikas et al. (2017) [1], Rezanejad et al. (2017) [5], Elhanafi et al. (2017) [9] e Connell et al. (2018) [10].

Como no presente trabalho utiliza-se dois fluidos diversos, leva-se em consideração o uso do modelo multifásico VOF (Volume of Fluid) para o tratamento da fase água e ar [11]. Destacam-se alguns autores que também utilizam a mesma metodologia para a solução dos problemas envolvendo energia das ondas Lisboa et al. (2016) [12], Gomes et al. (2017) [13, 14].

Por se tratar de um trabalho envolvendo simulação numérica, utiliza-se o recurso computacional de softwares específicos para dinâmica de fluidos computacional, em especial os baseados no Método dos Volumes Finitos (MVF), através do software FLUENT® (2009) [15]. O domínio computacional considerado avaliou um dispositivo CAO inserido em um tanque de ondas computacional.

Assim, o presente trabalho leva em consideração uma geometria bidimensional, em que se estuda as variações dos graus de liberdade $H_{l} / L$ (razão entre altura e largura da câmara hidropneumática), $H_{3}$ (submersão do dispositivo) e $L / l$ (razão entre largura da câmara hidropneumática e duto de passagem de ar). Verifica-se como a descontinuidade geométrica influencia na análise transiente da pressão e da vazão mássica de ar.

\section{MATERIAL E MÉTODOS}

\subsection{Dispositivo CAO}

Segundo Tolmasquim (2016) [2], o dispositivo CAO foi um dos primeiros conceitos em extração da energia das ondas do mar. Os critérios de classificação dos dispositivos levam em consideração dois fatores: localização de instalação e método de conversão de energia [16].

Em relação a localização dos dispositivos existem três diferentes classificações: quando o dispositivo encontra-se na costa o mesmo é chamado de onshore, quando encontra-se próximo à costa, chama-se nearshore e quando está afastado da costa chama-se offshore [17]. Quando se leva em consideração o princípio de funcionamento, tem-se: dispositivos CAO, corpos oscilantes (Absorção pontual) e dispositivos de galgamento [18].

Os dispositivos CAO são estruturas que possuem seu interior aberto para entrada e saída da água e encontram-se parcialmente submersos como mostra a Figura 1.

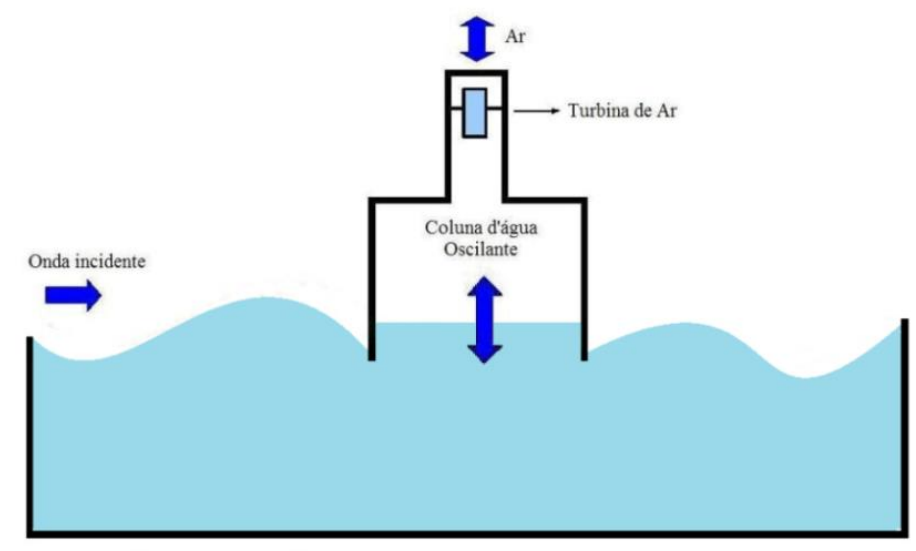

Figura 1: Funcionamento de um dispositivo CAO. 
Segundo Cruz e Sarmento (2004) [17], a conversão da energia das ondas em energia elétrica segue duas etapas: compressão e descompressão, sendo o processo de compressão quando a onda incide sobre o dispositivo e a descompressão quando a onda passa pelo dispositivo fazendo o ar retornar pela chaminé.

Em função do sentido de escoamento do ar variar conforme a fase, a turbina utilizada para conversão é do tipo Wells, em que a mesma independe do sentido de escoamento do ar e assim converte a energia cinética em mecânica.

\subsection{Representação Computacional}

O trabalho realizado foi desenvolvido de forma bidimensional, assim foi considerado um dispositivo CAO e um tanque de ondas associados, de forma que o conversor encontra-se inserido no tanque de ondas, a Figura 2 apresenta, de forma esquematizada, o dispositivo e o tanque.

Neste trabalho é considerada uma onda regular com características bem definidas e constantes: período $T=5 \mathrm{~s}$, comprimento $\lambda=37,6 \mathrm{~m}$ e profundidade de propagação $h=10 \mathrm{~m}$. Além disso, as constantes: comprimento do tanque de ondas $\left(C_{T}\right)$ e altura do mesmo são $188 \mathrm{~m}$ e $13 \mathrm{~m}$, respectivamente. As demais dimensões do domínio computacional apresentado na Figura 2 são indicadas na sequência do texto

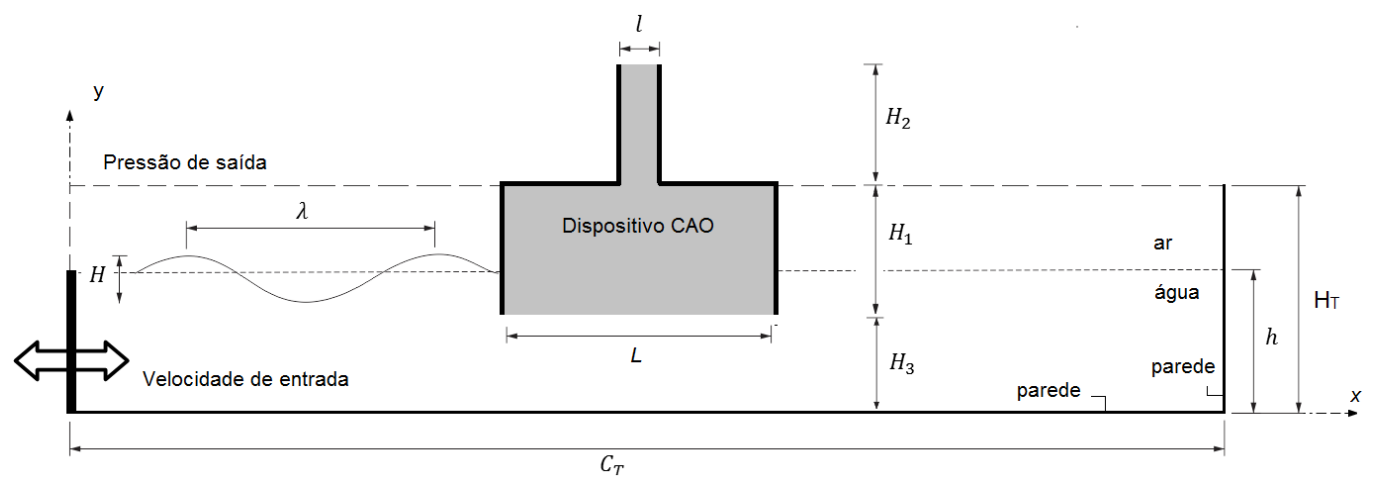

Figura 2: Representação esquemática do domínio computacional.

\subsection{Condições de Contorno}

Na Figura 2 pode-se verificar a geração da onda numérica a esquerda do tanque de ondas. A condição de contorno empregada é baseada na criação de uma função com as equações de velocidade inseridas numericamente como feito em Gomes et al. (2008) [19].

A aplicação da metodologia consiste em aplicar na região de geração da onda uma variação da velocidade de entrada (velocity inlet) através da função definida com as velocidades numéricas impostas. A utilização desta técnica resulta na separação do perfil de velocidade em componentes horizontais e verticais, com base na Teoria de Stokes de $2^{\mathrm{a}}$ ordem [6]. Assim, as Equações $1-2$ definem a condição de contorno apresentada:

$$
\begin{aligned}
& u=\frac{H}{2} g k \frac{\cosh (k z+k h)}{\omega \cosh (k h)} \cos (k x-\omega t)+\frac{3}{4}\left(\frac{H}{2}\right)^{2} \omega k \frac{\cosh 2 k(k+z)}{\sinh ^{4}(k h)} \cos 2(k x-\omega t) \\
& w=\frac{H}{2} g k \frac{\operatorname{senh}(k z+k h)}{\omega \cosh (k h)} \operatorname{sen}(k x-\omega t)+\frac{3}{4}\left(\frac{H}{2}\right)^{2} \omega k \frac{\operatorname{senh}^{2} k(k+z)}{\operatorname{senh}^{4}(k h)} \operatorname{sen} 2(k x-\omega t)
\end{aligned}
$$


em que: $H$ é a altura da onda incidente $(\mathrm{m}) ; g$ é a aceleração da gravidade $\left(\mathrm{m} / \mathrm{s}^{2}\right) ; k\left(\mathrm{~m}^{-1}\right)$ é o número de onda, $h$ é a profundidade (m), $T$ é o período de onda (s), $\omega(\mathrm{rad} / \mathrm{s})$ é a frequência, $x$ é a coordenada que representa a direção principal (m), $t$ é o tempo (s) e $z$ é a coordenada normal (m).

Como pode ser observado na Figura 2, as outras condições de contorno são definidas da seguinte maneira: na parte superior da superfície lateral esquerda, bem como na superfície superior do tanque, é aplicada uma condição de contorno de pressão atmosférica. Nas demais paredes que definem o tanque de ondas são aplicadas uma condição de parede, isto é, não deslizamento e impermeabilidade. Todo estudo de escoamentos de fluidos deve iniciar com uma condição inicia, este não é diferente, aplica-se uma condição inicial considerado que o fluido está em repouso.

\subsection{Geração da Malha Computacional}

A malha utilizada no presente trabalho foi construída através da metodologia stretched, essa estratégia adota a definição de regiões mais refinadas que outras, sendo estas regiões de interesse da investigação como a superfície livre [20].

A Figura 3 apresenta o esquema de divisão do domínio computacional de forma a subdividir as regiões de interesse para geração da malha refinada em diferentes partes.

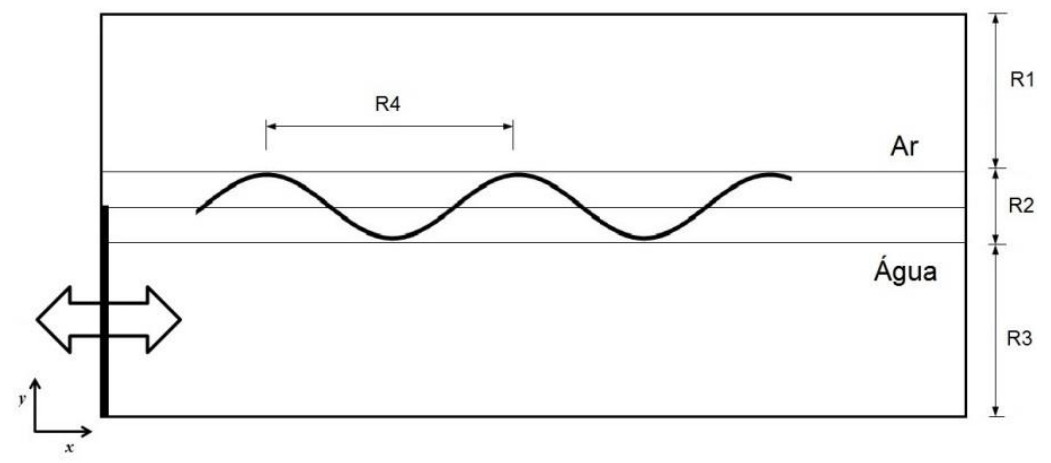

Figura 3: Malha definida através do número de volumes por característica da onda.

As divisões apresentadas na Figura 3 representam as três regiões de interesse da malha numérica, denominadas: $R_{l}, R_{2}$ e $R_{3}$. A região onde ocorre o desenvolvimento da onda, isto é, onde se encontram suas cavas e cristas (região $R 2$ ) adota-se um refinamento com 40 volumes na direção $y$ (o tamanho desse intervalo é equivalente a $H / 20$ ) e com 50 volumes por comprimento de onda, totalizando 250 volumes, recomendação descrita no trabalho de Gomes (2014) [6].

As outras regiões também são de interesse da simulação numérica, mas para os resultados esperados, não há a necessidade de um maior refinamento da malha. Assim, são utilizadas para as regiões $R I$ e $R 3$ um refinamento de 10 e 90 volumes, respectivamente [21]. A medida $R 4$ refere-se ao comprimento de onda.

\subsection{Modelo de Tratamento de Fluidos Imiscíveis}

A necessidade do tratamento de dois fluidos se faz presente na investigação em questão, uma vez que se verifica a interação entre água e ar. Para o estudo da associação dos fluidos, utiliza-se o método Volume of Fluid- VOF [11].

Nesta formulação, todas as fases são definidas e o volume ocupado por uma fase não pode ser ocupado por outra fase. O equacionamento do modelo utilizado é composto pela equação de conservação de massa com duas fazes (ar e água):

$$
\frac{\partial \rho}{\partial t}+\nabla \cdot(\rho \vec{v})=0
$$

A equação da conservação de quantidade de movimento: 
$\frac{\partial}{\partial t}(\rho \vec{v})+\nabla \cdot(\rho \vec{v} \vec{v})=-\nabla p+\nabla(\mu \bar{\tau})+\rho g$

E a equação da fração volumétrica:

$\frac{\partial(\alpha)}{\partial t}+\nabla \cdot(\alpha \vec{v})=0$

em que : $\rho$ é a massa específica do fluído $\left(\mathrm{kg} / \mathrm{m}^{3}\right), t$ é o tempo (s), $\vec{v}$ é o vetor velocidade do fluído $(\mathrm{m} / \mathrm{s}), p$ é a pressão estática $\left(\mathrm{N} / \mathrm{m}^{2}\right), \mu$ é a viscosidade $(\mathrm{kg} / \mathrm{m} . \mathrm{s}), \stackrel{\bar{\tau}}{\tau}$ é o tensor das tensões $\left(\mathrm{N} / \mathrm{m}^{2}\right)$ e $\vec{g}$ é a aceleração da gravidade $\left(\mathrm{m} / \mathrm{s}^{2}\right)$.

Por apresentar dois fluidos de trabalho, utiliza-se a fração de volume $(\alpha)$ para cálculos referentes a ocupação dos mesmos em determinadas alocações quando define-se numericamente o trabalho. Neste caso $\alpha_{\mathrm{ar}}=1-\alpha_{\text {água }}$, contém a interface entre água e ar. As que possuem $\alpha_{\text {água }}=0$ estão sem água e completas de ar $\left(\alpha_{\mathrm{ar}}=1\right)$; e, por sua vez, as que apresentam $\alpha_{\mathrm{ar}}=0$ estão cheias de água $\left(\alpha_{\text {água }}=1\right)$.

As médias dos valores para massa específica e viscosidade devem ser calculadas, pois as equações apresentadas anteriormente são resolvidas para a interação ar e água [22].

$$
\begin{aligned}
& \rho=\alpha_{\text {agua }} \rho_{\text {água }}+\alpha_{\text {ar }} \rho_{\text {ar }} \\
& \mu=\alpha_{\text {agua }} \mu_{\text {água }}+\alpha_{a r} \mu_{\text {ar }}
\end{aligned}
$$

\subsection{Arranjo Computacional}

Quando um trabalho computacional é realizado, define-se uma sequência de parâmetros que devem ser alocadas no software em uso para a solução do problema. Para resolução das equações de conservação de massa e quantidade de movimento utiliza-se o Método dos Volumes Finitos (MVF).

O uso do esquema de advecção upwind de primeira ordem para tratamento dos termos advectivos e a discretização espacial para a pressão foi realizado através do método PRESTO (Pressure Staggering Option) [24].

O método GEO-RECONSTRUCTION foi utilizado para a fração volumétrica. Para o acoplamento pressão-velocidade foi empregado o método PISO (Pressure Implicit eith Splitting of Operators) [23, 24].

A verificação da onda numérica é realizada para testar o modelo VOF. Assim, compara-se o resultado analítico com o numérico através da equação que define analiticamente a onda segue:

$$
\eta(x, t)=A \cos (k x-\omega t)+\frac{A^{2} k \cosh (k h)}{4 \operatorname{senh}^{3}(k h)}[2+\cosh (2 k h)] \cos 2(k x-\omega t)
$$

onde: $A$ é a amplitude da onda (m), dado por $H / 2$, x é a posição (m), $t$ é a variação do tempo (s), $k$ e $\omega$ são o número de onda $\left(\mathrm{m}^{-1}\right)$ e a frequência da onda $(\mathrm{rad} / \mathrm{s})$, respectivamente. 


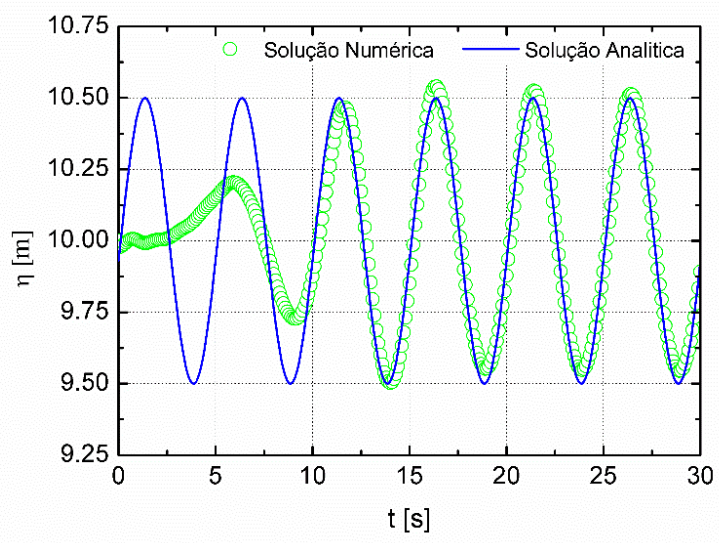

Figura 4: Comparação do resultado analítico e numérico da superfície livre.

A comparação dos resultados apresentados na Figura 4 indicam que as diferenças máximas obtidas na crista e na cava da onda são inferiores a 5\%; na média, e essa diferença diminui para aproximadamente $2 \%$ a medida que a onda se estabilizou.

\section{RESULTADOS}

Uma questão trabalhada na presente investigação refere-se ao fator de amplificação, ou seja, o efeito de pistão. São três os aspectos que influenciam no fator de amplificação: o comprimento da câmara CAO $(L)$, a altura da câmara CAO $\left(H_{l}\right)$ e a altura da onda incidente $(H)$.

Na Figura 5 é realizada uma comparação do comportamento transiente da elevação da superfície livre no interior da câmara CAO para três situações, considerando $H_{3}=9,50 \mathrm{~m}$ : em azul $-H_{1} / L=$ 0,0266 ( $L=37,6 \mathrm{~m}$ e $\left.H_{l}=1,0 \mathrm{~m}\right)$, em vermelho $-H_{l} / L=0,1346\left(L=16,7097 \mathrm{~m}\right.$ e $\left.H_{l}=2,2501 \mathrm{~m}\right)$ e em cinza $-H_{l} / L=1,0225\left(L=6,0637 \mathrm{~m}\right.$ e $\left.H_{l}=6,2007 \mathrm{~m}\right)$. Assim, dentre os casos estudados $H_{l} / L=$ 0,1346 foi considerado o caso ótimo, pois os picos de movimentação da superfície livre no interior da câmara CAO são maiores do que os atingidos quando $H_{1} / L=0,0266$. Para uma comparação entre os casos $H_{l} / L=0,1346$ e $H_{l} / L=1,0225$, verifica-se que não existe quase diferença para elevação da superfície livre.

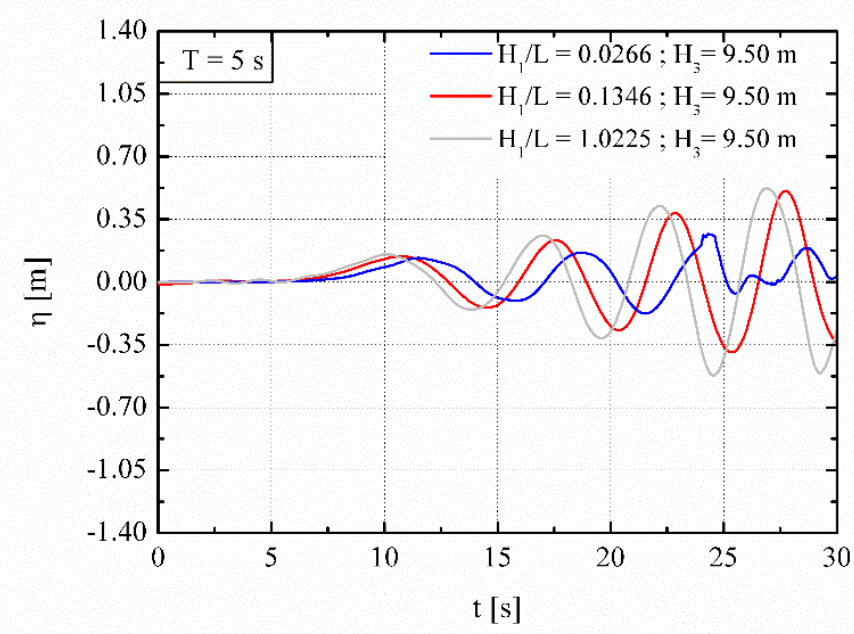

Figura 5: Análise transiente da elevação da superfície livre no interior da câmara CAO.

$\mathrm{Na}$ Tabela 1 são apresentados os valores para o volume de água e ar calculados. Com o aumento da relação $H_{I} / L$ observa-se, que o volume de ar no interior da câmara CAO aumenta e o volume de 
água diminui. Além disso, verifica-se que os volumes de água e ar se distribuem quase que igualmente em relação ao volume total da câmara CAO para o caso 2 .

Tabela 1: Influência da quantidade de água e ar no desempenho da câmara CAO.

\begin{tabular}{ccccccccc}
\hline Casos & $\mathbf{H}_{\mathbf{1}} / \mathbf{L}$ & $\mathbf{L} / \boldsymbol{\lambda}$ & $\mathbf{H} \mathbf{H} / \mathbf{H}$ & $\begin{array}{c}\mathbf{H}_{\mathbf{4}} \\
{[\mathbf{m}]}\end{array}$ & $\begin{array}{c}\mathbf{V}_{\mathbf{A R}} \\
{\left[\mathbf{m}^{\mathbf{3}}\right]}\end{array}$ & $\begin{array}{c}\mathbf{V}_{\text {ÁGUA }} \\
{\left[\mathbf{m}^{\mathbf{3}}\right]}\end{array}$ & $\begin{array}{c}\text { Pressão } \\
\mathbf{R M S}[\mathbf{P a}]\end{array}$ & $\begin{array}{c}\dot{\mathbf{m}} \mathbf{R M S} \\
{[\mathbf{k g} / \mathbf{s}]}\end{array}$ \\
\hline 1 & 0,0266 & 1,00 & 1,00 & 0,28 & 8,27 & 29,32 & 18,09 & 6,33 \\
2 & 0,1346 & 0,44 & 2,25 & 0,50 & 20,88 & 16,70 & 28,66 & 15,22 \\
3 & 1,0225 & 0,16 & 6,20 & 0,51 & 31,47 & 6,12 & 17,20 & 8,66 \\
\hline
\end{tabular}

O equacionamento para pressão, vazão mássica e potência hidropneumática podem ser verificados em Gomes (2014) [6]. Na Figura 6(a) é apresentada uma comparação do comportamento transiente da pressão quando $H_{3}=9,50 \mathrm{~m} \mathrm{e} H_{1} / L=0,1346$ (melhor caso) e quando $H_{3}=9,00 \mathrm{~m} \mathrm{e} H_{1} / L=0,0266$ (pior caso). No melhor caso geométrico, a distribuição de pressões é simétrica e suave, fato que deve ser levado em conta num projeto futuro da turbina de ar. Por outro lado, no pior caso, existe muita perturbação no campo de pressões, apresentando um comportamento assimétrico e instável.

Um dos motivos para essa instabilidade é à descontinuidade geométrica entre a câmara $\mathrm{CAO}$ e o duto da chaminé da câmara CAO, medida através do parâmetro $L / l$. A Figura $6(\mathrm{~b})$ apresenta a mesma análise, mas para a vazão mássica. Assim como para a pressão, o comportamento da vazão mássica no melhor caso é simétrico e suave e tem picos maiores em relação ao pior caso.

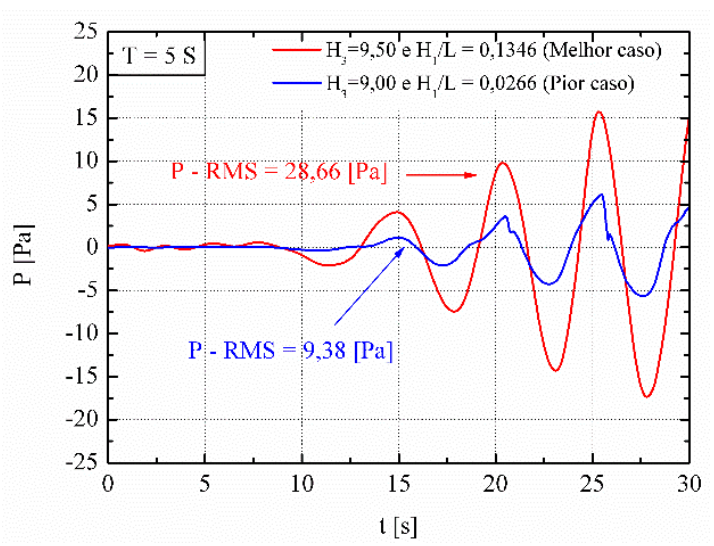

(a)

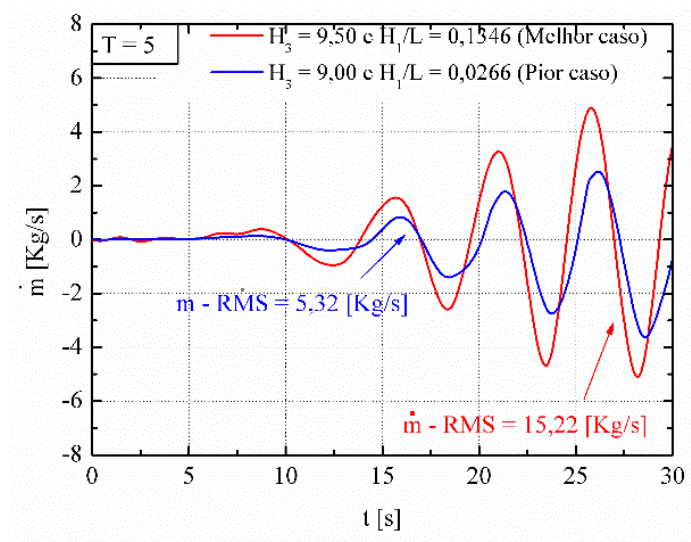

(b)

Figura 6: Comportamento transiente da (a) pressão e (b) vazão mássica.

Durante toda a simulação, a quantidade de fluido que escoa e transpassa o duto superior da câmara hidropneumática do dispositivo CAO é de aproximadamente $7.000 \mathrm{~kg}$ para o pior caso, enquanto que, no melhor caso, tem-se o valor de cerca $22.000 \mathrm{~kg}$ de ar escoam pela chaminé. Portanto, outra relação que deve ser investigada é a razão entre o comprimento da câmara do dispositivo CAO $(L)$ e o diâmetro da chaminé de saída da câmara CAO $(l)$. Através da relação $L / l$ é possível dimensionar a "descontinuidade geométrica" entre a câmara e a chaminé do dispositivo CAO. Esta relação influencia na perda de carga ao longo do escoamento.

Na Figura 7 é apresentado o comportamento da perda localizada para a variação de $H_{l} / L$ considerando $H_{3}=9,50 \mathrm{~m}$. Para tanto é considerado o primeiro período de incidência da onda no dispositivo $\mathrm{CAO}$, caracterizando na primeira metade do período uma contração $(l / L)$ e na segunda parte uma expansão $(L / l)$. Na contração destaca-se que, a partir de $l / L=0,075$ à medida que esta razão tende a 1, a perda localizada diminui (vide linha azul). E o mesmo pode ser verificado para a expansão a partir de $L / l=9,20$. Onde a medida que $L / l$ tende a 1 , a perda localizada diminui. 


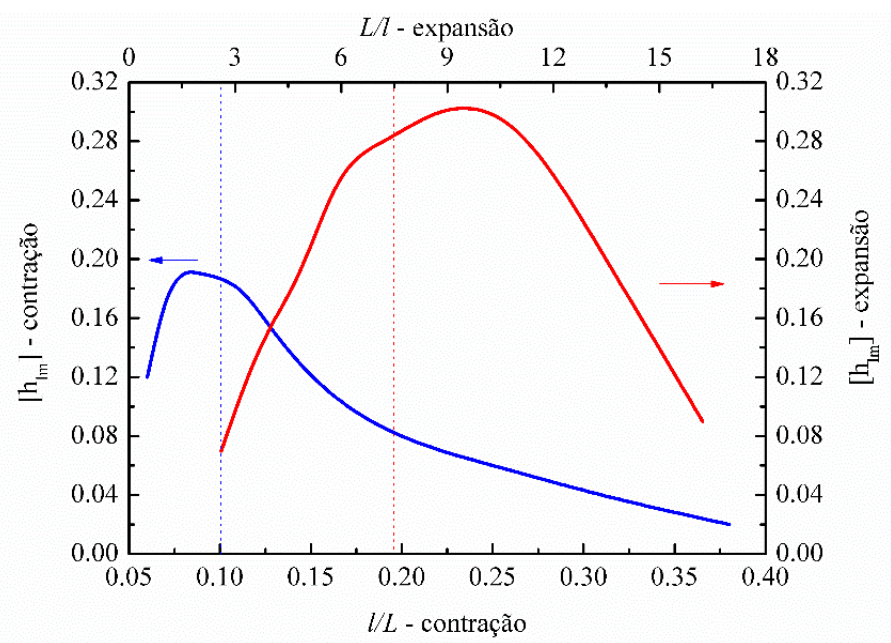

Figura 7: Análise da perda localizada para a variação de $H_{1} / L$ considerando $H_{3}=9,50 \mathrm{~m}$.

A investigação do efeito de $L / l$ no campo de pressões na câmara CAO se fez através da análise transiente da pressão para os três primeiros casos da variação de $H_{1} / L$ quando $H_{3}=9,50 \mathrm{~m}$, como pode ser observado na Figura 8.
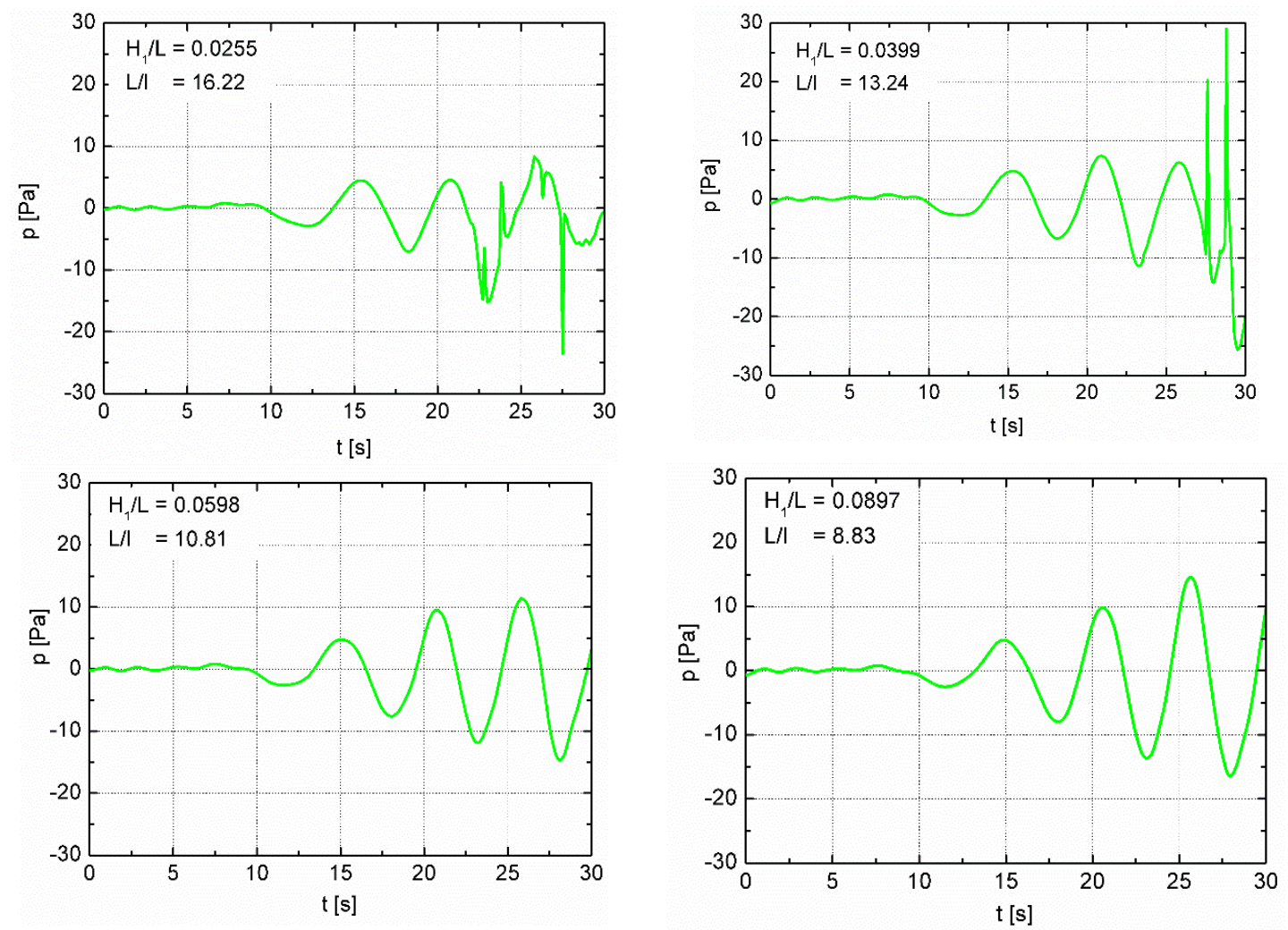

Figura 8: Comportamento transiente da pressão para a variação de $H_{l} / L$ quando $H_{3}=9,50 \mathrm{~m}$.

Observa-se que à medida que $L / l$ diminui (por consequência a descontinuidade geométrica tende a diminuir) os picos negativos de pressão deixam de existir e a distribuição de pressões tende a ficar simétrica, e isso ocorre sempre quando $H_{l} / L$ tende a aumentar, em função que esses dois graus de liberdade influenciam, simultaneamente, na forma geométrica da câmara hidropneumática, influenciando diretamente a pressão. 


\section{CONCLUSÃO}

A análise e otimização geométrica foi o principal foco de pesquisa apresentado na presente investigação. $O$ trabalho se deteve em analisar a influência da variação geométrica para o estudo do efeito de pistão e as variações de pressão e vazão mássica em dispositivos CAO.

Para a modelagem computacional foi utilizado o modelo multifásico Volume of Fluid que é aplicado para representar a interação entre a água e o ar, sendo que a solução numérica baseada no Método de Volumes Finitos. Em todas as situações foi realizada a otimização geométrica de três graus de liberdade do sistema: $H_{l} / L$ (relação entre altura e comprimento da câmara CAO), $H_{3}$ (profundidade de submersão do dispositivo $\mathrm{CAO}$ ) e $L / l$ (relação entre largura da câmara hidropneumática e chaminé do dispositivo).

Verificou-se o comportamento da vazão mássica e pressão transiente quando aplicado os graus de liberdade envolvidos no problema e observou-se que à medida que $L / l$ diminui (por consequência a descontinuidade geométrica tende a diminuir) os picos negativos de pressão deixam de existir e a distribuição de pressões tende a ficar simétrica, e isso ocorre sempre quando $H_{l} / L$ tende a aumentar. Concluindo-se que as características do caso que apresenta o mais alto desempenho são: $H_{3}=9,50 \mathrm{~m}, H_{l} / L=0,0897$ e $L / l=8,83$.

\section{AGRADECIMENTOS}

Os autores agradecem a Universidade Federal do Rio Grande do Sul (UFRGS), Universidade Federal do Rio Grande (FURG) e o Instituto Federal do Paraná (IFPR). O presente trabalho foi realizado com apoio da Coordenação de Aperfeiçoamento de Pessoal de Nível Superior - Brasil (CAPES) - Código de Financiamento 001 e CNPq.

\section{REFERÊNCIAS BIBLIOGRÁFICAS}

1. Vyzikas T, Deshoulières S, Barton M, Giroux O, Greaves D, Simmonds D. Experimental investigation of diferente geometries of fixed oscillating water column devices. Renew Energ. 2017;(104):248-258, doi.org/10.1016/j.renene.2016.11.061

2. Tolmasquim A. Energia Renovável Hidráulica, Biomassa, Eólica, Solar, Oceânica. Brasil: Interciência; 2016. $452 \mathrm{p}$.

3. Drew B, Plummer A, Sahinkaya MN. A review of wave energy converter technology. J Power Energ. 2009;(223):887-902, doi.org/10.1243/09576509JPE782

4. Clément A. Wave energy in Europe: current status and perspectives. Renew Sust Energ Rev. 2002;(6):405-431, doi.org/10.1016/S1364-0321(02)00009-6

5. Rezanejad K, Soares CG, López I, Carballo R. Experimental and numerical investigation of the hydropneumatic performance of an oscillating water column wave energy converter. Renew Energ. 2017;(106):1-16.

6. Gomes MN. Constructal Design de Dispositivos Conversores de Energia das Ondas do Mar em Energia Elétrica do Tipo Coluna de Água Oscilante. [Tese]. Porto Alegre (BR): Universidade Federal do Rio Grande do Sul; 2014. 212 p.

7. Barbosa DVE. Análise de dispositivos de extração de energia das ondas do tipo galgamento. [dissertação]. Rio Grande (BR): Universidade Federal do Rio Grande; 2016. 98 p.

8. Martins J. Design Construtal de um dispositivo de galgamento onshore em escala real para diferentes áreas de construção e ondas monocromáticas. [dissertação]. Rio Grande (BR): Universidade Federal do Rio Grande; 2016. 110 p.

9. Elhanafi A, Macfarlane G, Fleming A, Leong Z. Experimental and numerical investigations on the hydropneumatic performance of a floating-moored oscillating water column wave energy converter. Appl Energ. 2017;(205):369-390, doi.org/ 10.1016/j.apenergy.2017.07.138

10. Connell KO, Thiebaut F, Kelly G, Cachman A. Development of a free heaving OWC model with nonlinear PTO interaction. Renew Energ. 2018;(177):108-115, doi.org/10.1016/j.renene.2017.10.027

11. Hirt CW, Nichols BD. Volume of fluid (VOF) method for the dynamics of free boundaries. J Comput Phys. 1981;(39):201-225, doi: 10.1016/0021-9991(81)90145-5.

12. Lisboa RC, Teixeira PRF, Didier E. Regular and irregular wave propagation analysis in a flume with numerical beach using a navier-stokes based model. Defect Diffus Forum. 1016;327:81-90. 
13. Gomes MN, Deus MJ, Santos ED, Isoldi LA, Rocha LAO. The choice of geometric constrains value applied in the constructal design of oscillating water column device, 2017. Proceedings of the XXXVIII Iberian Latin-American Congress on Computational Methods in Engineering, 2017 oct 22-25. Florianópolis, SC. p. 1-20.

14. Gomes MN, Lorenzini G, Rocha LAO, Santos ED, Isoldi LA. Constructal design applied to the geometric evaluation of an oscillating water column wave energy converter considering different real scale wave periods. J Eng Thermophys. 2017;27(2):173-190.

15. Fluent Inc. FLUENT 12.0 Theory Guide. Canonsburg:SouthPoint, 2009. 814 p.

16. Falcão A. Wave energy utilization: A review of the Technologies. Renew Sust Energ Rev. 2009;(14):899-918, doi: 10.1016/j.rser.2009.11.003.

17. Cruz JP, Sarmento AJ. Energia das Ondas: Introdução aos Aspectos Tecnológicos, Econômicos e Ambientais. Alfragide: Instituto do Ambiente, 2004. 65 p.

18. Teixeira PR, Davyt DP, Didier E, Ramalhais R. Numerical Simulation of an Oscillating Column Device Using a Code Base on Navier-Stokes Equations. Energ. 2013;(61):513-530, doi: 10.1016/j.energy.2013.08.062.

19. Gomes MN, Olinto CR, Rocha LA, Souza JA, Isoldi LA. Computacional Modeling of a Regular Wave Tank. Eng Térmica. 2009;(8):44-50, doi: 10.1109/MCSUL.2009.27.

20. Mavriplis DJ. Unstructured Grid Techniques. Ann Rev Fluid Mech. 1997;(29):473-514.

21. Barreiro TG. Estudo da interação de uma onda monocromática com um conversor de energia [dissertação]. Lisboa (PT): Faculdade de Ciências e Tecnologia da Universidade Nova de Lisboa; 2009.

22. Srinivasan AJ, Saito k. Modeling the disintegration of modulated liquid jets using volume-of-fluid (VOF) methodology. Appl Mathem Model. 2011 jan;08(35):3710-3730, doi.org/10.1016/j.apm.2011.01.040

23. Patankar SV. Numerical Heat Transfer and Fluid Flow. Estados Unidos: McGraw-Hill, 1980. 196 p.

24. Versteeg HK, Malalasekera W. An Introduction to Computational Fluid Dynamics, Malaysia: Pearson Press; 2007. $520 \mathrm{p}$. 